Check for updates

Cite this: DOI: 10.1039/c9ta10113f

\section{Local mobility in electrochemically inactive sodium in hard carbon anodes after the first cycle $\uparrow$}

\author{
Anders C. S. Jensen, (D) *ab Emilia Olsson, (D) abc Heather Au, ${ }^{\mathrm{b}}$ Hande Alptekin, ${ }^{\mathrm{b}}$ \\ Zhengqiang Yang, (D) a Stephen Cottrell, ${ }^{d}$ Koji Yokoyama, ${ }^{d}$ Qiong Cai, (D) c \\ Maria-Magdalena Titirici (D) ${ }^{b}$ and Alan J. Drew ${ }^{\star a}$
}

Sodium ion batteries are a promising alternative to current lithium ion battery technology, providing relatively high capacity and good cycling stability at low cost. Hard carbons are today the anodes of choice but they suffer from poor rate performance and low initial coulombic efficiency. To improve the understanding of the kinetics of sodium mobility in these materials, muon spin rotation spectroscopy and density functional theory calculations were used to probe the intrinsic diffusion of sodium in a characteristic hard carbon sample. This revealed that atomic diffusion between sites is comparable to that observed in transition metal oxide cathode materials in sodium ion batteries, suggesting that the poor rate performance is not limited by site-site jump diffusion rates. In addition, diffusion was observed in the sodium that is irreversibly stored during the first cycle, suggesting that some of these sodium atoms are not immobilised in the solid electrolyte interface (SEI) layer but are still blocked from long range diffusion, thereby rendering the sodium electrochemically inactive.
Received 12th September 2019 Accepted 10th December 2019

DOI: $10.1039 /$ c9ta10113f

rsc.li/materials-a
Structurally, $\mathrm{HC}$ is made of primarily $\mathrm{sp}^{2}$ hybridised carbon in a graphitic arrangement with extensive defects and a turbostratic stacking of nano-sized domains. ${ }^{6,7}$ The stacking of the graphitic domains results in nano-pores typically in the size of a few nanometers. ${ }^{8}$ Both the graphitic layers and the pores have been suggested to provide active sites for sodium storage, ${ }^{\mathbf{9 , 1 0}}$ but the kinetics of these processes are poorly understood.

The intrinsic diffusion dynamics of battery materials have been extensively studied using muon spin rotation ( $\mu \mathrm{SR})$ spectroscopy for lithium ion batteries ${ }^{11-13}$ and cathodes in sodium ion batteries, ${ }^{\mathbf{1 4}, 15}$ and have revealed activation energies for diffusion in the range of $225-571 \mathrm{meV}$ for sodium ions in transition metal oxides and sodium jump frequencies of $\sim 0.15-$ $0.2 \mu \mathrm{s}^{-1}$ at room temperature. Combining this with jump distances determined by PXRD, diffusion constants can be derived, which for $\mathrm{Na}_{x} \mathrm{CoO}_{2}$ are in the range $4 \times 10^{-11}$ to $5 \times$ $10^{-12} \mathrm{~cm}^{2} \mathrm{~s}^{-1}$ depending on $x \cdot{ }^{15} \mu \mathrm{SR}$ spectroscopy can therefore give direct information on the intrinsic diffusion constants independent of microstructure, particle size and various other factors that can affect electrochemical techniques. ${ }^{\mathbf{1 5}}$

To better understand the diffusion dynamics in HCs, $\mu$ SR spectroscopy was performed on a HC carbon sample after sodiation in the first cycle and after one full sodiation and desodiation cycle. This revealed a jump frequency of $\sim 0.2 \mu \mathrm{s}^{-1}$ at room temperature for the sodiated material, comparable with the above-mentioned cathode materials, and a lower activation energy of just 88(13) meV. In the desodiated sample the sodium still exhibited jump frequencies comparable with those of the sodiated sample, suggesting that the sodium is not 
immobilized in the SEI layer, but blocked from long range diffusion, while maintaining local mobility. Using an expanded graphite model, density functional theory (DFT) calculations suggested that the diffusion in HC primarily occurs between sites where sodium interacts mainly with one graphitic plane (in a graphene-like environment) and is slowed down in sites where the planes are too close together.

\section{Experimental}

\section{Synthesis}

The hard carbon material was produced by hydrothermal carbonization of glucose. In a typical batch, $10 \mathrm{~g}$ glucose (D(+)-glucose, $\geq 99.5 \%$, Sigma Aldrich) and $3.6 \mathrm{~g}$ citric acid ( $\geq 99.5 \%$, Sigma Aldrich) were dissolved in $90 \mathrm{~mL}$ deionised water. The solution was placed into a $200 \mathrm{~mL}$ autoclave reactor vessel and heated at $230{ }^{\circ} \mathrm{C}$ for 12 hours. After reaction, the resulting solid was separated from the supernatant and dried under vacuum at $80{ }^{\circ} \mathrm{C}$ overnight, before further carbonisation at $1300{ }^{\circ} \mathrm{C}$ for 2 hours under nitrogen atmosphere.

\section{Battery assembly}

Electrodes were prepared from slurries of $90 \mathrm{wt} \%$ hard carbon and $10 \mathrm{wt} \%$ sodium carboxymethyl cellulose binder $\left(M_{\mathrm{w}} \sim\right.$ 250000 , Sigma Aldrich) in water, applied in a $250 \mu \mathrm{m}$ layer on $\mathrm{Al}$ foil (conductive carbon coated, $18 \mu \mathrm{m}$, MTI corporation). The foils were cut to size (typically $4 \mathrm{~cm} \times 4 \mathrm{~cm}$ ) and dried under vacuum at $80{ }^{\circ} \mathrm{C}$ before being placed in the glovebox for final assembly. The electrolyte and cell preparation were conducted in an argon-filled glovebox with $\mathrm{H}_{2} \mathrm{O}<0.5 \mathrm{ppm}, \mathrm{O}_{2}<0.5 \mathrm{ppm}$. Sodium hexafluorophosphate $\left(\mathrm{NaPF}_{6}\right.$, Alfa Aesar, 99+\%) was first dried at $80{ }^{\circ} \mathrm{C}$ under vacuum for $16 \mathrm{~h}$. A $1: 1$ mixture, by volume, of ethylene carbonate (EC, anhydrous 99\%, Sigma Aldrich) and dimethyl carbonate (DMC, 99.9+\%, Sigma Aldrich) was dried over 4 Å molecular sieves (20 vol\%) for one day before use, and the solvent extracted from the top fraction to avoid contamination. A $1 \mathrm{M}$ electrolyte solution was prepared by stirring $\mathrm{NaPF}_{6}$ in $\mathrm{EC} / \mathrm{DMC}$ until fully dissolved. To assemble the cell, a carbon anode, glass fibre separator (Whatman GF/B glass microfibre) saturated with electrolyte, and sodium metal sheet (sodium ingot, 99.8\% metals basis, Alfa Aesar) were assembled inside a plastic pouch cell (Cookhouse) with aluminium current collector tabs (PI-KEM), before sealing. The finished batteries were left for a minimum of 1 hour to equilibrate. Electrochemical characterization was performed in a CR2032 coin cell.

\section{Battery cycling}

The battery cells were cycled at a current density of $30 \mathrm{~mA} \mathrm{~g}^{-1}$ (0.1C) using a BaSyTec CTS battery test system between $2.5 \mathrm{~V}$ and $0 \mathrm{~V}$ vs. $\mathrm{Na}^{+} / \mathrm{Na}$.

\section{Muon spectroscopy}

For each $\mu$ SR measurement eight $4 \times 4 \mathrm{~cm}$ pouch cells were used. The battery foils were extracted from the cells and the hard carbon material scraped off. The resulting powder was placed in a standard ISIS Ti cell. Each cell contained $\sim 0.1 \mathrm{~g}$ $\mathrm{cm}^{-2}$ of sample and additional $0.1 \mathrm{~g} \mathrm{~cm}^{-2}$ of Ti degrader to ensure the maximum concentration of muons stopping in the sample. All sample handling was conducted in a glovebox. $\mu \mathrm{SR}$ was performed at the EMU instrument ${ }^{16}$ at ISIS (Harwell, UK), ${ }^{17}$ using a CCR cryostat to control temperature in the range of 50$400 \mathrm{~K}^{12,18}$

\section{Powder X-ray diffraction (PXRD)}

PXRD was performed on a Xenoc nano-inXider using a $\mathrm{Cu} \mathrm{K}_{\alpha}$ source and a two-detector setup for simultaneous small angle Xray scattering (SAXS)/wide angle X-ray scattering (WAXS) measurements.

\section{Computational method}

For the atomic scale simulations of $\mathrm{Na}$ incorporation and migration in expanded graphite, all calculations were made using DFT as implemented in the Vienna $A b$ initio Simulation Package (VASP, version 5.3.5). ${ }^{19-22}$ The DFT calculations utilized the projector-augmented wave method (PAW) to describe the ion-electron interaction, ${ }^{23}$ and the plane wave cut-off and $k$ space integrals were chosen so that the total energy was converged to $1 \mathrm{meV}$ per atom. The kinetic energy cut-off was $800 \mathrm{eV}$, with a $5 \times 5 \times 2 \Gamma$-centred Monkhorst-Pack grid to sample the Brillouin zone. ${ }^{24}$ To express the interacting electron exchange-correlation energy (with electronic convergence criteria of $10^{-5} \mathrm{eV}$ and ionic convergence criteria of $10^{-3} \mathrm{eV}$ $\AA^{-1}$ ), the generalized gradient approximation (GGA) with Perdew-Burke-Ernzerhof (PBE) ${ }^{25,26}$ functionals were used. All calculations were performed spin-polarized. ${ }^{27}$ Due to the large polarizability of the graphite and sodium, ${ }^{28}$ it is important to include dispersion corrections to accurately estimate the metal adsorption strength, and interlayer binding energy. ${ }^{29-31}$ The van der Waals forces in between the graphene sheets in graphite have been accounted for by different functionals and dispersion corrections. This selection is here made based on the interlayer binding energy, and lattice parameters. After testing of different correction schemes based on cell optimisations of graphene, AA and AB stacked graphite bulks, the DFT-D3 method with BeckeJohnson damping by Grimme and co-workers was selected. This setup was previously used to study $\mathrm{Na}$ adsorption and migration on graphene, and has been verified in the literature. ${ }^{28,32,33}$

Metal migration energy barrier calculations were conducted with the climbing image nudged elastic band method (CI-NEB), as implemented in VASP. The metal migration paths were constructed using the VASP Transition State Tools. ${ }^{34}$ From the CI-NEB calculations, the metal migration energy barrier is then taken as the maximum energy difference between the initial and final states on the migration path. ${ }^{35,36}$

\section{Results and discussion}

The hard carbon sample was synthesised by the HTC process ${ }^{37}$ and treated at $1300{ }^{\circ} \mathrm{C}$ under nitrogen. Structural characterisation was done using combined SAXS and WAXS (Fig. 1). The SAXS region (Fig. 1A) showed a typical Guinier-Porod shape with an initial slope at low Q resulting from surface scattering 

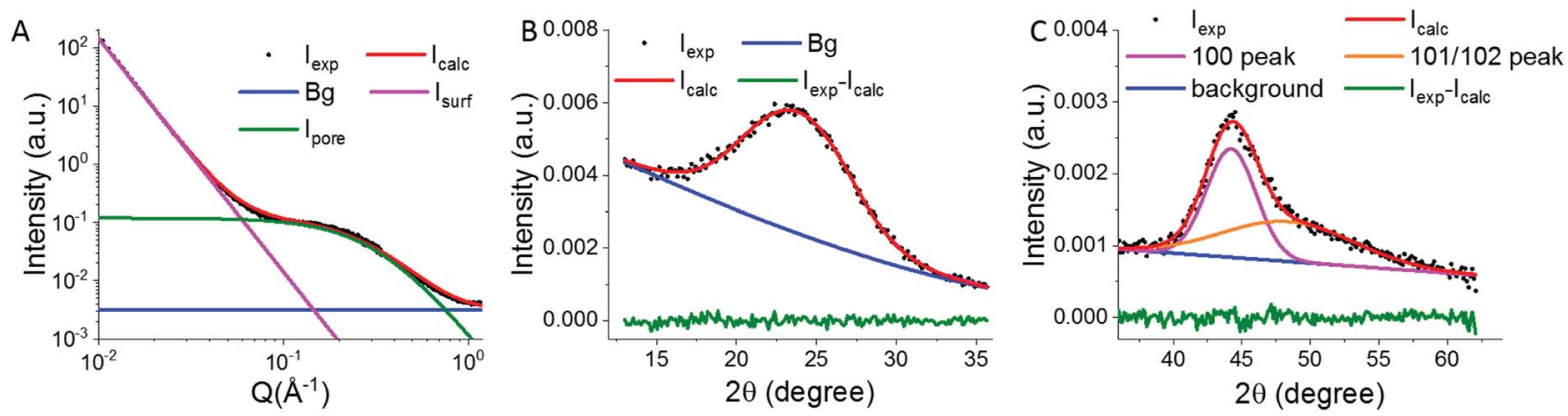

Fig. 1 (A) SAXS result for the anode material and associated fit using the Guinier-Porod model. ${ }^{8}$ (B) The 002 peak of the hard carbon related to the stacking of the graphitic planes. (C) The 100 peak related to the in plane order of the graphitic basal planes, overlapping with the $101 / 102$ peaks. All the peaks in the WAXS range was fitted using Gaussian peak shapes.

and a Guinier plateau and Porod slope originating from pore scattering. ${ }^{8}$ This could be fitted with a simple model $^{8}$ from which the pore size could be determined to be 19(3) A. The WAXS region showed two main peaks, the 002 peak at $\sim 25^{\circ}$ and the 100 peak at $45^{\circ}$ (Fig. $1 \mathrm{~B}$ and C). From the position of the 002 peak the interlayer distance was determined to be 3.745(8) A. Using the width of the two peaks and the Scherrer equation the size and thickness of the stacks of graphitic domains was esti-

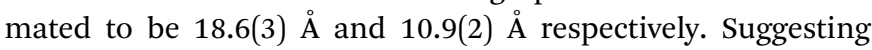
basal planes with roughly 8 aromatic rings across the plane and 3-4 layer thick stacks, consistent with the general characteristic of most hard carbons. ${ }^{5,8}$ During battery cycling, the material showed a reasonable reversible capacity of $222 \mathrm{~mA} \mathrm{~h}^{-1}$ with an initial coulombic efficiency of $81 \%$ (Fig. 2A). The charge/ discharge curves showed both a sloping region between 1 and $0.1 \mathrm{~V}$ and plateau region below $0.1 \mathrm{~V}$, suggesting sodium incorporation via different mechanisms in the two voltage regions (Fig. 2A). These regions have previously been attributed to intercalation between graphitic planes ${ }^{9}$ during the sloping region and as quasi-metallic clusters forming in the nanopores $^{\mathbf{8 , 1 0}}$ during the plateau region. Additionally, the sample shows relatively poor rate capability as expected from a hard carbon sample,${ }^{5}$ retaining only $10 \%$ of the capacity at $2 \mathrm{C}$ relative to $0.1 \mathrm{C}$ (Fig. 2B).

Two samples were chosen to investigate sodium diffusion. The first sample was a fully sodiated material discharged at $0.1 \mathrm{C}$ and contained sodium that contributes to both the reversible and irreversible capacity. The second sample was fully sodiated and subsequently desodiated at the same current density and as
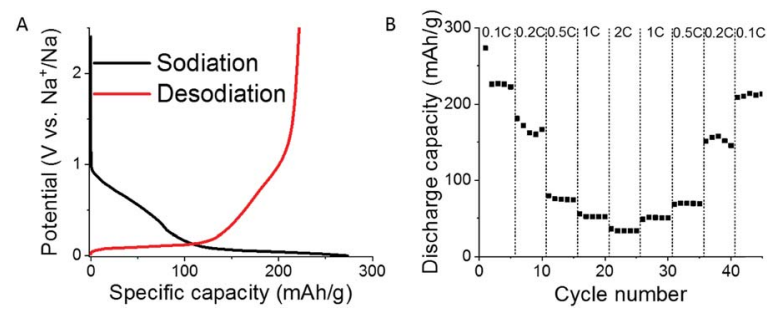

Fig. 2 (A) Charge(desodiation)/discharge(sodiation) curves for the sample at $0.1 \mathrm{C}$ with $\mathrm{Na}$ metal counter electrode, and discharge capacity at various charging rates (B). such only contained sodium contributing to the irreversible capacity. The muon spectroscopy measurements were done in transverse field mode (Fig. 3), instead of the conventional zero and longitudinal field mode, as disordered carbons often have complex signals in these modes from defect sites that are decoupled in transverse field mode. ${ }^{38}$ The asymmetry function (A) was modelled using a two component model with a rotating field $(F)$ and a dampening function $(G)$ available in the WIMDA software package: ${ }^{39}$

$$
A=F \times G
$$

with

$$
F(B, \phi, t)=\cos \left(\gamma_{\mu} B t+\phi\right)
$$

where $B$ is the external field, $t$ is time, $\phi$ the phase and $\gamma_{\mu}$ the gyromagnetic ratio of the muon. The diffusion constants were extracted from the dampening of the rotating field using the Abragam equation: ${ }^{\mathbf{1 2 , 1 8 , 4 0}}$

$$
G\left(\sigma, \tau_{\mathrm{Na}}, t\right)=\exp \left(-2 \sigma^{2} \tau_{\mathrm{Na}}^{2}\left(\exp \left(-\frac{t}{\tau_{\mathrm{Na}}}\right)-1+\frac{t}{\tau_{\mathrm{Na}}}\right)\right)
$$

with $\sigma$ being the dampening frequency in the static regime caused by the internal field and $1 / \tau_{\mathrm{Na}}$ the frequency of the intermittent field interpreted as the jump frequency of sodium. $\sigma$ was determine by cooling the sample to $50 \mathrm{~K}$ where all diffusion events are assumed to be frozen out (Fig. 3A). For all other temperatures $\sigma$ was fixed to determine $1 / \tau_{\mathrm{Na}}$ (example fit shown in Fig. 3B).

The jump frequency $\left(1 / \tau_{\mathrm{Na}}\right)$ shows an onset temperature of diffusion of around $210 \mathrm{~K}$ for the sodiated sample and a subsequent increase consistent with an Arrhenius behaviour and an activation energy of $88(13) \mathrm{meV}$ (Fig. 3B). While the jump frequency represents an average of both the reversibly and irreversibly bound sodium, it is dominated by the former as this represents $80 \%$ of the total sodium content. The large scatter of points at high temperature (Fig. 3B and C) is caused by the low dampening of the asymmetry function (Fig. 3A) making accurate determination of $1 / \tau_{\mathrm{Na}}$ above $0.3 \mu \mathrm{s}^{-1}$ difficult. In the desodiated material the sodium present was bound irreversibly (Fig. 2A), suggesting that it was immobilized in the SEI layer or 

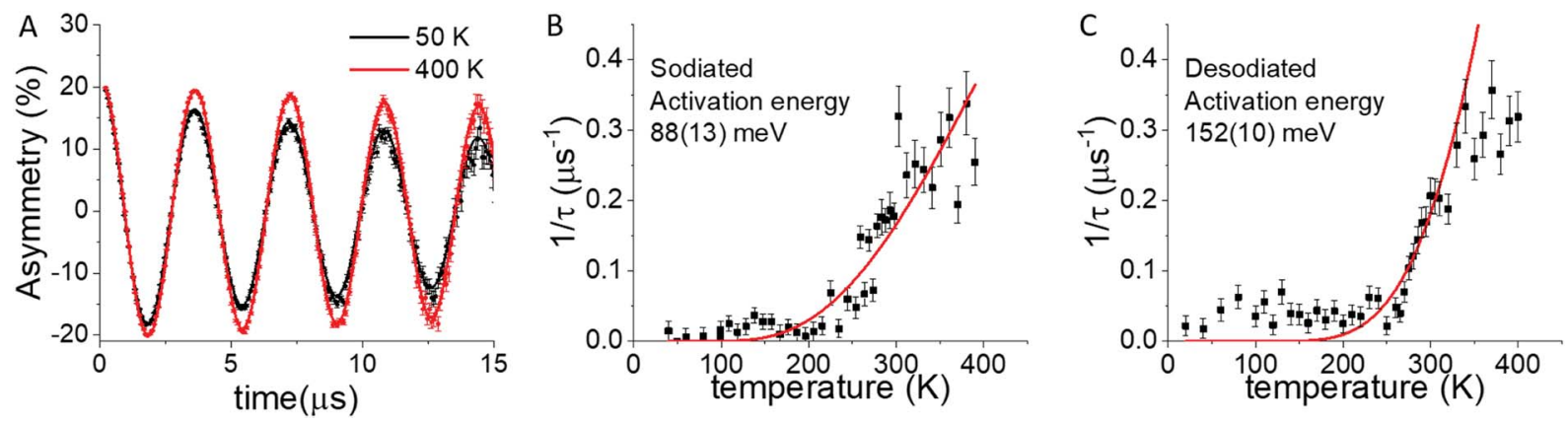

Fig. 3 (A) Example muon spectrum measured in transverse field mode at $50 \mathrm{~K}$ (black), where the sodium is assumed static, and at $400 \mathrm{~K}$ (red), where the $\mathrm{Na}$ is undergoing diffusion. Points represent the measured values and lines the fit using eqn (1). From the muon spectra, the jump frequency of $\mathrm{Na}$ determined for a sodiated sample (B) and a sample that has undergone sodiation and subsequent desodiation (C). Red lines in (B) and (C) represent estimates of the jump frequency using the Arrhenius equation. Arrhenius plots are shown in Fig. S1. $\dagger$

at strongly bound sites in the carbon structure. However, sodium diffusion is still observed, but with an onset temperature around $250 \mathrm{~K}$ and a higher activation energy of $152(10) \mathrm{meV}$ (Fig. 3C). Additionally, a sharper transition to a dynamic regime is observed, suggesting that the sodium left after desodiation is mainly associated with a particular site or a few similar sites. The higher activation energy could be consistent with sodium bound at a defect site, which has been suggested to have higher binding affinity compared to sites in expanded defect-free graphite. ${ }^{33}$ Despite the higher activation energy, the sodium should still be mobile ${ }^{\mathbf{4 1}}$ and able to partake in electrochemical reactions given the relatively low activation energy compared to that observed for cathode materials. ${ }^{\mathbf{1 4 , 1 5}}$ This indicates that the sodium is not only immobilized at the surface, but rather blocked from long range diffusion while maintaining local mobility. Therefore, whilst the sodium is not immobilised within the SEI layer, this same layer may block off some of the diffusion paths connecting the pores and the graphitic layers with the particle surface. This is also seen by the very similar jump frequency for both samples at room temperature of around $0.2 \mu \mathrm{s}^{-1}$. Indicating that the diffusion is not limited by the site-site jump kinetics but rather the availability of long range diffusion paths which is not only sensitive to the synthesis procedure but also the side reactions occurring during cycling, as seen by the mobility of the electrochemically inactive sodium observed in the desodiated sample. These results are consistent with previous studies in which the capacity loss was reduced by limiting surface reactions in a soft/ hard carbon composite, effectively protecting the diffusion paths from being blocked during the first cycle. ${ }^{5}$

\section{Modelling of sodium storage and migration}

To investigate sodium migration in the expanded graphitic layers identified above, DFT simulations of a $4 \times 4 \times 2$ supercell (64 atoms) of two graphitic layers with $\mathrm{AB}$ layer ordering and the experimentally determined $3.75 \AA$ interlayer distance (Fig. 1) was performed. The binding energy for interlayer distances between 3.3-4 Å was also calculated (Fig. S2 $\dagger$ ) and showed that the sodium intercalation for a single sodium atom is energetically favourable above $3.5 \AA$. A cell optimization of the $a$ and $b$ lattice vectors was performed whilst keeping the interlayer distance $(c)$ constant before investigating metal intercalation and migration, whereby all lattice parameters are kept fixed in geometry optimizations for simulating the sodium binding in the expanded graphitic layers.

To form an understanding of the energetic favourability of sodium intercalation in the expanded graphitic layers, the intercalation energy $\left(E_{\text {int }}\right)$ of a single sodium ion in the two expanded graphitic layers was calculated (eqn (4)). ${ }^{\mathbf{2}}$

$$
E_{\mathrm{int}}=E_{\mathrm{Na}-\mathrm{GL}}-\mu_{\mathrm{A}}-E_{\mathrm{gl}}
$$

$E_{\mathrm{Na}-\mathrm{GL}}$ is the total energy of the two graphitic layers with sodium, $E_{\mathrm{gl}}$ is the total energy of the two graphitic layers only, and $\mu_{\mathrm{A}}$ is the sodium chemical potential. ${ }^{33}$ The calculated intercalation energy was found to be $-0.81 \mathrm{eV}$, corresponding to the site above the centre of the aromatic ring in the basal plane. This can be compared to the $E_{\text {int }}$ for $\mathrm{Na}$ in pristine graphite $(c=3.35$ A) which was calculated to be $0.51 \mathrm{eV}$. Hence, in agreement with the experimental results presented above, sodium storage and intercalation is energetically stable in the graphitic layers with an interlayer distance of $3.75 \AA$ A. The binding energies of up to 8 sodium atoms were also calculated and are shown in Fig. S2. $\dagger$ This showed a decrease in the energy with increasing sodiation, but also showed an increase in the interlayer spacing not observed during cycling of hard carbons. ${ }^{9,43}$ This suggests that the intercalation composition does not exceed $\mathrm{NaC}_{32}$ and as such can only account for $\sim 70 \mathrm{~mA} \mathrm{~h}^{-1}$ of the specific capacity.

Using the above model, the sodium ion migration energy barrier is calculated using the CI-NEB method. Two migration paths were investigated (Fig. 4B), sodium migrating over a $\mathrm{C}-\mathrm{C}$ bond (Path 1), and over a carbon atom in one layer and above a $\mathrm{C}_{6}$-ring in the other layer (Path 2). The resulting energy migration barriers are calculated to be $0.33 \mathrm{eV}$ for Path 1, and $0.18 \mathrm{eV}$ for Path 2. Hence, Path 2 is the more energetically favourable sodium migration pathway. It is important to note, however, that these migration energy barriers are both lower than $0.5 \mathrm{eV}$, which is considered to be the cut-off energy for metal diffusion at room temperature. ${ }^{41}$ Hence, sodium diffusion in the expanded graphitic layers is expected to take place through both pathways in hard carbon, with pathway 2 being 


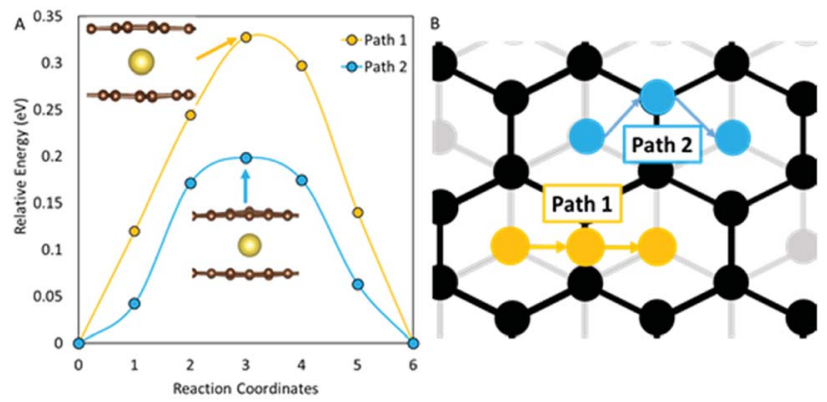

Fig. 4 (A) Calculated migration barriers for sodium diffusion in the expanded graphitic layers, with two different pathways shown in (B). Path 1 involves sodium migrating over a $\mathrm{C}-\mathrm{C}$ bond, and Path 2 over a carbon atom. Yellow and blue circles in (B) represent a sodium ion at different sites of the migration pathways; the black and grey circles are carbon atoms in alternating graphitic layers. The inset in (A) shows the side view of the transition states for the two pathways (i.e. the structure for reaction coordinate 3 ). For these, brown spheres are carbon and yellow spheres sodium.

more prominent. In both diffusion pathways the transition state is associated with a small distortion of the carbon layer in agreement with the Arrhenius behaviour observed from the muon data (Fig. 3B, C and S1 $\dagger$ ), which suggests that the diffusion is phonon mediated, indicating that phonons locally distort the basal planes, lowering the energy barrier to diffusion. Furthermore, comparing these migration barriers to sodium on graphene $(0.10 \mathrm{eV}),{ }^{33}$ which could be used as a proxy for the carbon basal planes in hard carbons, it is clear that sodium migration barriers in the expanded graphitic layers are higher. Comparing these migration barriers to the experimental average of sodium diffusion barriers $(0.088 \mathrm{eV})$ suggests that sodium diffusion in the sodiated HC mainly occurs where the sodium ions primarily interact with a single graphitic layer, whereas diffusion in the desodiated sample (Fig. 3C) has an activation energy comparable with Path 2 (Fig. 4B), suggesting that the irreversible capacity loss may be concentrated in sites where the sodium interacts equally with two graphitic planes or, as mentioned previously, with defect sites. ${ }^{33}$ A more detailed discussion of the effect of the graphitic interlayer distance and morphology on metal diffusion will be discussed in a future publication.

Combining the results from the electrochemistry, PXRD, muon spectroscopy, and DFT simulations, two characteristic sodium sites can be described: a high mobility site with high reversibility, and a low mobility site with limited reversibility (Fig. 5), consistent with the intercalation (low mobility) and pore filling (high mobility) model proposed in literature. ${ }^{8-10}$ The high mobility site was observed to have a lower activation barrier to diffusion using both DFT and muon spectroscopy and constitutes part of the reversible capacity mainly observed in the plateau region (Fig. 2A). The DFT model suggests that the sodium in this site is mainly interacting with a single graphitic sheet, suggesting that it is associated with the internal surface of the nanopores. The sodium associated with low mobility sites, on the other hand, has a high energy barrier to diffusion as observed from muon spectroscopy and DFT modelling, and

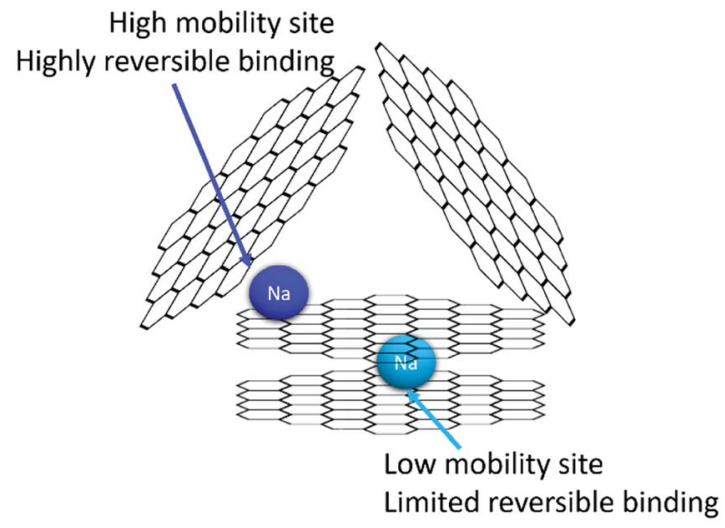

Fig. 5 Schematic of the intercalation and pore filling mechanism proposed in literature, showing the high mobility site likely located in the pores with the sodium interacting with a single graphitic layer, and the low mobility site of intercalation with limited reversible capacity.

is related to part of the irreversible capacity accounting for the remaining $20 \%$ of the sodium. DFT modelling suggests that this site is mainly associated with intercalated sodium or sodium in defects sites. ${ }^{33}$ While this site is not electrochemically active, it does still show local mobility, but is somehow blocked from long-range diffusion.

\section{Conclusions}

Muon spectroscopy revealed that sodium diffusion in hard carbon initiates at temperatures as low as $210 \mathrm{~K}$ with an activation energy of $88(13) \mathrm{meV}$ for a fully sodiated sample. DFT calculations further suggest that the diffusion primarily occurs along surface sites of the graphitic domains, assumed to be associated with the internal surfaces of the pores. The irreversibly bound sodium may be concentrated in sites where the sodium interacts equally with two graphitic domains (i.e. intercalation sites), or at defect sites. After desodiation, the remaining sodium is not immobilized entirely in the SEI layer as expected but exhibits local mobility without long range diffusion, possibly due to the available diffusion pathways being blocked off by side reactions during battery cycling.

\section{Conflicts of interest}

There are no conflicts to declare.

\section{Acknowledgements}

The authors would like to thank Dr Gavin Stenning for help on the Nano InXider instrument in the Materials Characterization Laboratory at the ISIS Neutron and Muon Source. E. O., Q. C., A. J. D. and M. M. T. would like to thank Engineering and Physical Sciences Research Council (EP/R021554/1). E. O. would like to acknowledge the use of Athena at HPC Midlands+, which was funded by the EPSRC under grant EP/P020232/1, and the Eureka HPC cluster at the University of Surrey. We are grateful to the 
UK Materials and Molecular Modelling Hub for computational resources, which is partially funded by EPSRC (EP/P020194/1).

\section{Notes and references}

$1 \mathrm{~T}$. Liu and X. Li, Biomass-derived nanostructured porous carbons for sodium ion batteries: a review, Mater. Technol., 2019, 34(4), 232-245.

2 L. Fan, Q. Liu, S. Chen, Z. Xu and B. Lu, Soft Carbon as Anode for High-Performance Sodium-Based Dual Ion Full Battery, Adv. Energy Mater., 2017, 7(14), 1602778.

3 Q. Wang, C. Zhao, Y. Lu, Y. Li, Y. Zheng, Y. Qi, X. Rong, L. Jiang, X. Qi, Y. Shao, D. Pan, B. Li, Y.-S. Hu and L. Chen, Advanced Nanostructured Anode Materials for Sodium-Ion Batteries, Small, 2017, 13(42), 1701835.

4 M.-M. Titirici, R. J. White, C. Falco and M. Sevilla, Black perspectives for a green future: hydrothermal carbons for environment protection and energy storage, Energy Environ. Sci., 2012, 5(5), 6796-6822.

5 F. Xie, Z. Xu, A. C. S. Jensen, H. Au, Y. Lu, V. Araullo-Peters, A. J. Drew, Y.-S. Hu and M.-M. Titirici, Hard-Soft Carbon Composite Anodes with Synergistic Sodium Storage Performance, Adv. Funct. Mater., 2019, 1901072.

6 S. F. Parker, S. Imberti, S. K. Callear and P. W. Albers, Structural and spectroscopic studies of a commercial glassy carbon, Chem. Phys., 2013, 427, 44-48.

7 K. Jurkiewicz, S. Duber, H. E. Fischer and A. Burian, Modelling of glass-like carbon structure and its experimental verification by neutron and X-ray diffraction, J. Appl. Crystallogr., 2017, 50(1), 36-48.

8 D. A. Stevens and J. R. Dahn, An In Situ Small-Angle X-Ray Scattering Study of Sodium Insertion into a Nanoporous Carbon Anode Material within an Operating Electrochemical Cell, J. Electrochem. Soc., 2000, 147(12), 4428-4431.

9 J. K. Mathiesen, R. Väli, M. Härmas, E. Lust, J. Fold von Bülow, K. M. Ø. Jensen and P. Norby, Following the inplane disorder of sodiated hard carbon through operando total scattering, J. Mater. Chem. A, 2019, 7(19), 11709-11717.

10 J. M. Stratford, P. K. Allan, O. Pecher, P. A. Chater and C. P. Grey, Mechanistic insights into sodium storage in hard carbon anodes using local structure probes, Chem. Commun., 2016, 52(84), 12430-12433.

11 I. Umegaki, S. Kawauchi, H. Sawada, H. Nozaki, Y. Higuchi, K. Miwa, Y. Kondo, M. Månsson, M. Telling, F. C. Coomer, S. P. Cottrell, T. Sasaki, T. Kobayashi and J. Sugiyama, Liion diffusion in $\mathrm{Li}$ intercalated graphite $\mathrm{C}_{6} \mathrm{Li}$ and $\mathrm{C}_{12} \mathrm{Li}$ probed by $\mu+$ SR, Phys. Chem. Chem. Phys., 2017, 19(29), 19058-19066.

12 J. Sugiyama, H. Nozaki, I. Umegaki, K. Mukai, K. Miwa, S. Shiraki, T. Hitosugi, A. Suter, T. Prokscha, Z. Salman, J. S. Lord and M. Månsson, Li-ion diffusion in $\mathrm{Li}_{4} \mathrm{Ti}_{5} \mathrm{O}_{12}$ and $\mathrm{LiT}_{2} \mathrm{O}_{4}$ battery materials detected by muon spin spectroscopy, Phys. Rev. B: Condens. Matter Mater. Phys., 2015, 92(1), 014417.

13 I. D. Johnson, T. E. Ashton, E. Blagovidova, G. J. Smales, M. Lübke, P. J. Baker, S. A. Corr and J. A. Darr,
Mechanistic insights of $\mathrm{Li}^{+}$diffusion within doped $\mathrm{LiFePO}_{4}$ from Muon Spectroscopy, Sci. Rep., 2018, 8(1), 4114.

14 M. Månsson, I. Umegaki, H. Nozaki, Y. Higuchi, I. Kawasaki, I. Watanabe, H. Sakurai and J. Sugiyama, Na-ion dynamics in Quasi-1D compound $\mathrm{NaV}_{2} \mathrm{O}_{4}$, J. Phys.: Conf. Ser., 2014, 551, 012035.

15 M. Månsson and J. Sugiyama, Muon-spin relaxation study on Li- and Na-diffusion in solids, Phys. Scr., 2013, 88(6), 068509.

16 S. R. Giblin, S. P. Cottrell, P. J. C. King, S. Tomlinson, S. J. S. Jago, L. J. Randall, M. J. Roberts, J. Norris, S. Howarth, Q. B. Mutamba, N. J. Rhodes and F. A. Akeroyd, Optimising a muon spectrometer for measurements at the ISIS pulsed muon source, Nucl. Instrum. Methods Phys. Res., Sect. A, 2014, 751, 70-78.

17 A. Drew, K. Yokoyama and A. C. S. Jensen, The effect of porosity on sodium diffusion in hard carbons for $\mathrm{Na}$ ion batteries (NIB), ed. S. I. N. a. M. Source, 2019.

18 E. Karlsson, The use of positive muons in metal physics, Phys. Rep., 1982, 82(5), 271-338.

19 G. Kresse and J. Furthmüller, Efficiency of ab-initio total energy calculations for metals and semiconductors using a plane-wave basis set, Comput. Mater. Sci., 1996, 6(1), 15-50.

20 G. Kresse and J. Furthmüller, Efficient iterative schemes for ab initio total-energy calculations using a plane-wave basis set, Phys. Rev. B: Condens. Matter Mater. Phys., 1996, 54(16), 11169-11186.

21 G. Kresse and J. Hafner, Ab initio molecular-dynamics simulation of the liquid-metal-amorphous-semiconductor transition in germanium, Phys. Rev. B: Condens. Matter Mater. Phys., 1994, 49(20), 14251-14269.

22 G. Kresse and J. Hafner, Ab initio molecular dynamics for liquid metals, Phys. Rev. B: Condens. Matter Mater. Phys., 1993, 47(1), 558-561.

23 P. E. Blöchl, Projector augmented-wave method, Phys. Rev. B: Condens. Matter Mater. Phys., 1994, 50(24), 17953-17979.

24 H. J. Monkhorst and J. D. Pack, Special points for Brillouinzone integrations, Phys. Rev. B: Solid State, 1976, 13(12), 5188-5192.

25 J. P. Perdew, K. Burke and M. Ernzerhof, Generalized Gradient Approximation Made Simple, Phys. Rev. Lett., 1996, 77(18), 3865-3868.

26 J. P. Perdew, K. Burke and M. Ernzerhof, Generalized Gradient Approximation Made Simple [Phys. Rev. Lett. 77, 3865 (1996)], Phys. Rev. Lett., 1997, 78(7), 1396.

27 Y. Ma, P. O. Lehtinen, A. S. Foster and R. M. Nieminen, Magnetic properties of vacancies in graphene and singlewalled carbon nanotubes, New J. Phys., 2004, 6, 68.

28 X. Fan, W. T. Zheng and J.-L. Kuo, Adsorption and Diffusion of Li on Pristine and Defective Graphene, ACS Appl. Mater. Interfaces, 2012, 4(5), 2432-2438.

29 X. Fan, W. T. Zheng, J.-L. Kuo and D. J. Singh, Adsorption of Single $\mathrm{Li}$ and the Formation of Small Li Clusters on Graphene for the Anode of Lithium-Ion Batteries, ACS Appl. Mater. Interfaces, 2013, 5(16), 7793-7797.

30 M. Amft, S. Lebègue, O. Eriksson and N. V. Skorodumova, Adsorption of $\mathrm{Cu}, \mathrm{Ag}$, and $\mathrm{Au}$ atoms on graphene 
including van der Waals interactions, J. Phys.: Condens. Matter, 2011, 23(39), 395001.

31 S. Lee, M. Lee, H. Choi, D. S. Yoo and Y.-C. Chung, Effect of nitrogen induced defects in $\mathrm{Li}$ dispersed graphene on hydrogen storage, Int. J. Hydrogen Energy, 2013, 38(11), 4611-4617.

32 S. Thinius, M. M. Islam, P. Heitjans and T. Bredow, Theoretical Study of Li Migration in Lithium-Graphite Intercalation Compounds with Dispersion-Corrected DFT Methods, J. Phys. Chem. C, 2014, 118(5), 2273-2280.

33 E. Olsson, G. Chai, M. Dove and Q. Cai, Adsorption and migration of alkali metals ( $\mathrm{Li}, \mathrm{Na}$, and $\mathrm{K}$ ) on pristine and defective graphene surfaces, Nanoscale, 2019, 11(12), 52745284.

34 G. Henkelman, VTST Tools, accessed August, 2018.

35 G. Henkelman, B. P. Uberuaga and H. Jónsson, A climbing image nudged elastic band method for finding saddle points and minimum energy paths, J. Chem. Phys., 2000, 113(22), 9901-9904.

36 G. Henkelman and H. Jónsson, Improved tangent estimate in the nudged elastic band method for finding minimum energy paths and saddle points, J. Chem. Phys., 2000, 113(22), 9978-9985.
37 M.-M. Titirici, M. Antonietti and N. Baccile, Hydrothermal carbon from biomass: a comparison of the local structure from poly- to monosaccharides and pentoses/hexoses, Green Chem., 2008, 10(11), 1204-1212.

38 M. Riccò, D. Pontiroli, M. Mazzani, M. Choucair, J. A. Stride and O. V. Yazyev, Muons Probe Strong Hydrogen Interactions with Defective Graphene, Nano Lett., 2011, 11(11), 4919-4922.

39 F. L. Pratt, WIMDA: a muon data analysis program for the Windows PC, Phys. Rev. B: Condens. Matter Mater. Phys., 2000, 289-290, 710-714.

40 A. Abragam and A. Abragam, The principles of nuclear magnetism, Oxford University Press, 1961.

$41 \mathrm{~K}$. Nakada and A. Ishii, Migration of adatom adsorption on graphene using DFT calculation, Solid State Commun., 2011, 151(1), 13-16.

42 S. B. Zhang and J. E. Northrup, Chemical potential dependence of defect formation energies in GaAs: Application to Ga self-diffusion, Phys. Rev. Lett., 1991, 67(17), 2339-2342.

43 D. A. Stevens and J. R. Dahn, The Mechanisms of Lithium and Sodium Insertion in Carbon Materials, J. Electrochem. Soc., 2001, 148(8), A803-A811. 